Supporting Information for

From Organized High-throughput Data to Phenomenological Theory using Machine Learning: The Example of Dielectric Breakdown

Chiho Kim, ${ }^{1}$ Ghanshyam Pilania, ${ }^{2}$ and Ramamurthy Ramprasad ${ }^{*}, 1$

${ }^{1}$ Department of Materials Science \& Engineering, and Institute of Materials Science, University of Connecticut, 97 North Eagleville Road, Storrs, Connecticut 06269-3136, USA

${ }^{2}$ Materials Science and Technology Division, Los Alamos National Laboratory, Los Alamos, New Mexico 87545, USA

*E-mail: rampi@uconn.edu

S1: Breakdown mechanism

S2: Phenomenological model discovery work flow

S3: Statistical learning methods

S4: Computed intrinsic dielectric breakdown field and primary features

S5: Error analysis for prediction models

S6: $\Omega$-dimensional features with prediction errors

S7: Prediction models found by LASSO-LSF with 1-dimensional compound features

S8: Dataset used for validation of prediction models

References 


\section{S1: BREAKDOWN MECHANISM}

The electrons in the conduction band can gain energy due to an external electric field and lose energy due to electron-phonon scattering as shown in Figure S1a. Under the influence of a sufficiently large electric field, the energy of the conduction electrons can quickly reach the impact ionization threshold, leading to dielectric breakdown. ${ }^{1-4}$ The rate of the electron-phonon scattering tends to follow the electron density of states (DOS) for the conduction band (see Figures S1b and S1c for C and Si, respectively). Both gain and loss of the energy are related to the scattering rate, but only the energy gain is electric field dependent, as shown in Figure S1d and S1e, for C and Si, respectively.
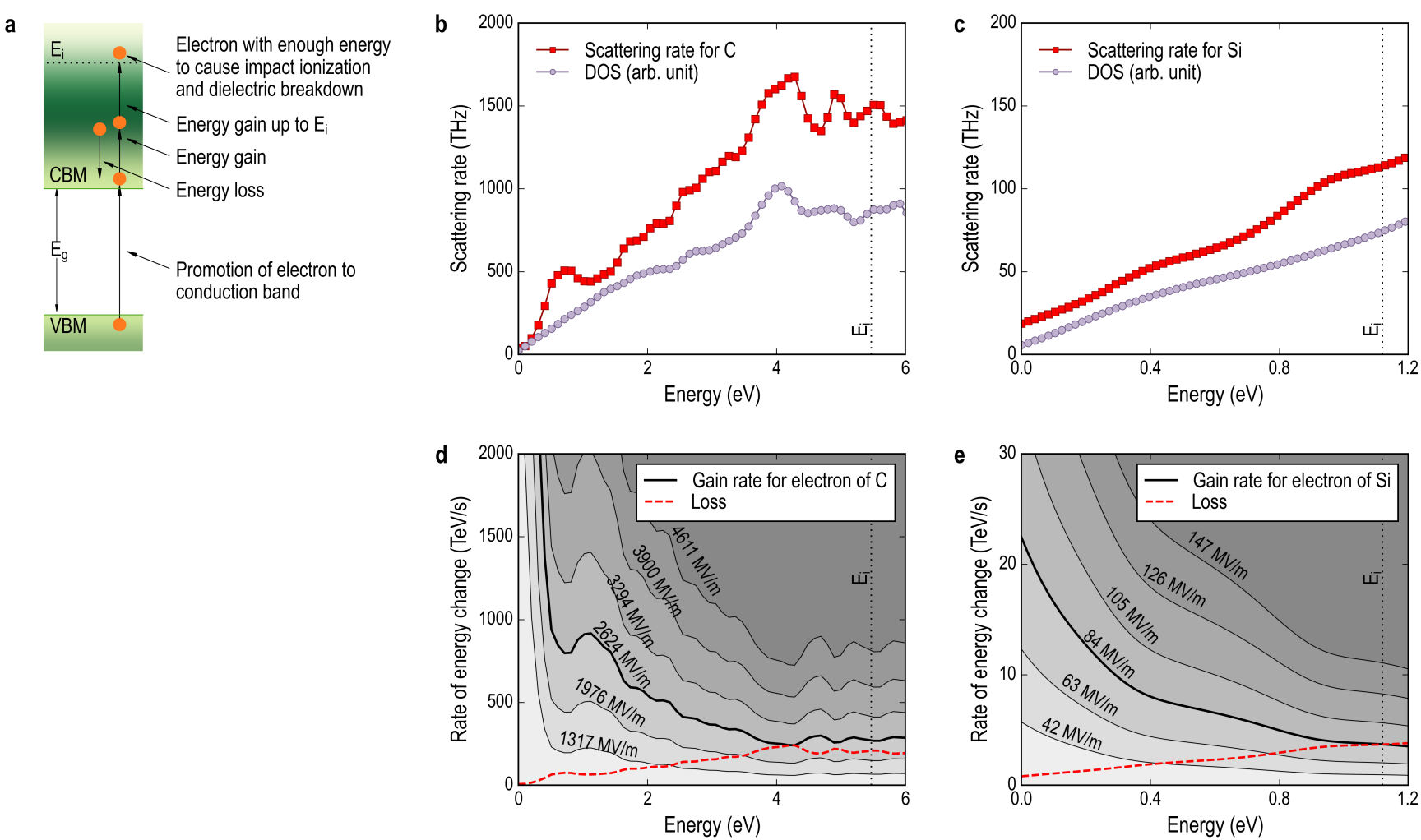

Figure S1. Mechanism of intrinsic dielectric breakdown of the insulating materials. (a) Schematic illustration of behavior of electrons under the electric field. The electrons can gain the energy from the external electric field, and lose the energy by electron-phonon scattering. (b) Electron-phonon scattering rate and DOS of C for the energy range from conduction band minimum $(C B M=0 \mathrm{eV})$ to impact ionization threshold energy $\left(E_{\mathrm{i}}=5.5 \mathrm{eV}\right)$. (c) Scattering rate and DOS of Si $\left(E_{\mathrm{i}}=1.1 \mathrm{eV}\right)$. (d) and (e) Rate of energy change of the electron due to the external electric field (gain) and electron-phonon scattering (loss) for $\mathrm{C}$ and $\mathrm{Si}$, respectively. 


\section{S2: PHENOMENOLOGICAL MODEL DISCOVERY WORKFLOW}

A detailed model discovery workflow for the intrinsic dielectric breakdown field is shown in Figure S2. As the first step, the intrinsic dielectric breakdown field for each of the 82 dielectric materials was computed using expensive quantum mechanical calculations. After generating a validated dataset of the intrinsic dielectric breakdown field, a set of 8 primary features was generated. These features when combined with 12 prototypical functions resulted in 96 features of one functions, 4,480 features of two functions and 183,368 features of three functions, giving a total of 187,944 compound features. While primary features were directly used in the kernel ridge regression (KRR), ${ }^{5-7}$ random-forest regression (RFR), ${ }^{5}$ least absolute shrinkage and selection operator (LASSO) $)^{5,8}$ based down-selection was first employed on the 187,952 (sum of 8 primary and 187,944 compound) features to down-select 36 most relevant ones. These 36 compound features were then subjected to a linear least square fit model to discover functional relationship with the intrinsic dielectric breakdown field. It is interesting to note that all three cross-validated learning models point towards the same two properties, namely, $E_{\mathrm{g}}$ and $\omega_{\max }$, as the most relevant descriptors to predict the intrinsic dielectric breakdown field.

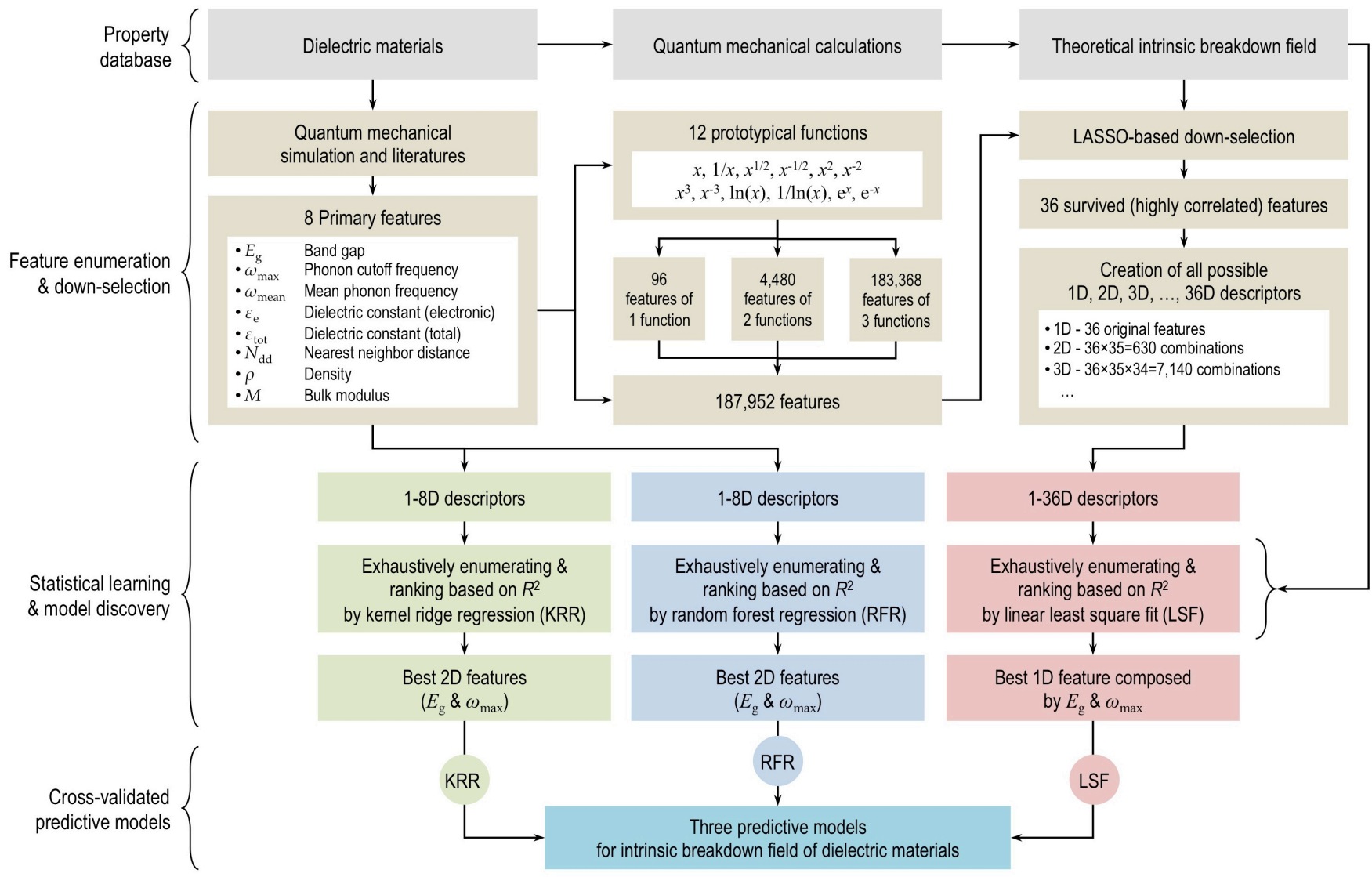

Figure S2. Detailed workflow used for data-driven discovery of a phenomenological model of intrinsic dielectric breakdown field. 


\section{S3: STATISTICAL LEARNING METHODS}

To establish a mapping between the computed breakdown field and the feature space we used three different learning schemes, as follows.

\section{Kernel Ridge Regression (KRR)}

Within the KRR similarity-based learning model, the estimate of a property, $P_{j}^{\text {Est }}$ of a new system $j$ (say, in the test or validation set) is given by a sum of weighted kernel functions (here taken to be Gaussians) over the entire training set, as

$$
P_{j}^{\text {Est }}=\sum_{i=1}^{N} \alpha_{i} \exp \left(-\frac{1}{2 \sigma^{2}}\left|\mathbf{F}^{i j}\right|^{2}\right)
$$

where $i$ runs over the systems in the training dataset, and $\left|\mathbf{F}^{i j}\right|=\left|\mathbf{d}_{i}-\mathbf{d}_{j}\right|$, the Euclidean distance between the feature vectors $\mathbf{d}_{i}$ and $\mathbf{d}_{j}$. The coefficients $\alpha_{i}$ s and the parameter $\sigma$ are obtained from the training (or learning) process built on minimizing the expression $\sum_{i=1}^{N}\left(P_{i}^{\text {Est }}-P_{i}^{\text {DFT }}\right)^{2}+\lambda \sum_{i=1}^{N} \alpha_{i}^{2}$ with $P_{i}^{\text {Est }}$ being the estimated property value, $P_{i}^{\text {DFT }}$ the DFT value, and $\lambda$ a regularization parameter. The explicit solution to this minimization problem is $\boldsymbol{\alpha}=(\mathbf{K}+\lambda \mathbf{I})^{-1} \mathbf{P}^{\text {DFT }}$, where $\mathbf{I}$ is the identity matrix, and $K_{i j}=\exp \left(-\frac{1}{2 \sigma^{2}}\left|\boldsymbol{F}^{i j}\right|^{2}\right)$ is the kernel matrix elements of all materials in the training set. The parameters $\lambda$ and $\sigma$ are determined in an inner loop of fivefold cross validation using a logarithmically scaled fine grid.

\section{Random-forest Regression (RFR)}

The RFR method is based on an ensemble of unpruned regression trees. These decision trees are created by using bootstrap samples of the training data, consisting of some or all primary features along with the target property. A large ensemble of decision trees is built by randomly selecting subsets of the selected features and the corresponding observations. There are two parameters in this method: number of trees and minimum node size. The number of trees should only be chosen to be sufficiently large to stabilize the test set error. In our case, we found that going beyond 20 trees did not improve the model performance on the test set predictions. However, we note that there is no penalty for having too many (more than minimally required) trees. ${ }^{9}$ This is in contrast to other machine learning algorithms which require a definite stopping rule to avoid overfitting. The second parameter, minimum node size, is arguably another tuning parameter, which determines the minimum size of nodes below which no split will be attempted. In our case, a value of 2 was used for this parameter. It has some effect on the size of the trees grown and its selection also depends on the size of the overall dataset that one is using to learn from. We find that the performance of RFR is relatively insensitive to changes in the minimum node size, as long as it is relatively small (between 1 and 3 ).

\section{Least absolute shrinkage and selection operator (LASSO)-based Model Selection}

Faced with a large set of features, our goal is to down-select to a small subset of those that gives the best linear fit to the target property. For a training set containing $N$ cases and the total number of features be- 
$\operatorname{ing} N_{\mathrm{f}}$, the LASSO-based feature selection works by minimizing $\sum_{i=1}^{N}\left(\beta_{0}+\sum_{j=1}^{N} C_{i j} \beta_{j}-P_{i}^{\text {DFT }}\right)^{2}+$ $\lambda^{\prime} \sum_{j=1}^{N_{\mathrm{f}}}\left|\beta_{j}\right|$, where $i$ and $j$ index the members of the training set and feature vector components, respectively, $C_{i j}$ is the value of the $j$ th component of the feature of case $i, \lambda^{\prime}$ is a regularization parameter, and $\beta_{j}$ is the coefficient of the $j$ feature component (with $\beta_{0}$ being the constant term in the linear fit). Systematically increasing $\lambda^{\prime}$ makes more and more feature coefficients to shrink exactly to zero, thereby identifying the most relevant features.

\section{S4: COMPUTED INTRINSIC DIELECTRIC BREAKDOWN FIELD AND PRIMARY FEATURES}

The dataset used in the present study consists of the intrinsic dielectric breakdown field computed within the framework of density functional theory (DFT), ${ }^{10}$ and 8 relatively easily accessible primary features for 82 model dielectric materials spanning a large chemical space. Except for the band gap (for which experimental values were available), the rest of the features were computed using DFT-based quantum mechanical simulations. This dataset, along with the ground state crystal structures of the compounds studied, is provided below in Table S1.

Table S1. List of 82 dielectric materials with their DFT-calculated intrinsic dielectric breakdown field and basic material properties. $\left(F_{\mathrm{b}}\right.$ : intrinsic dielectric breakdown field in $\mathrm{MV} / \mathrm{m}, E_{\mathrm{g}}$ : experimental band gap in $\mathrm{eV}, \omega_{\max }$ : phonon cutoff frequency in $\mathrm{THz}, \omega_{\text {mean }}$ : mean phonon frequency in $\mathrm{THz}, \varepsilon_{\mathrm{e}}$ : electronic contribution of dielectric constant, $\varepsilon_{\text {tot }}$ : total dielectric constant, $d_{\mathrm{NN}}$ : nearest neighbor distance in $\AA, \rho$ : density in $\mathrm{g} / \mathrm{cm}^{3}, M$ : bulk modulus in GPa, RS: rock salt, $\mathrm{CC}$ : cesium chloride, ZB: zinc blende)

\begin{tabular}{|c|c|c|c|c|c|c|c|c|c|c|}
\hline Name & $F_{\mathrm{b}}$ & $E_{\mathrm{g}}$ & $\omega_{\max }$ & $\omega_{\text {mean }}$ & $\varepsilon_{\mathrm{e}}$ & $\varepsilon_{\text {tot }}$ & $d_{\mathrm{NN}}$ & $\rho$ & $M$ & Structure \\
\hline \multicolumn{11}{|c|}{ Alkali metal halides } \\
\hline $\mathrm{LiF}$ & 4829.6 & $13.6(11)$ & 19.599 & 10.435 & 2.068 & 7.936 & 1.943 & 2.938 & 86.582 & $\mathrm{RS}$ \\
\hline $\mathrm{LiCl}$ & 679.8 & $9.4(11)$ & 12.531 & 6.301 & 3.150 & 10.003 & 2.477 & 2.317 & 40.537 & $\mathrm{RS}$ \\
\hline $\mathrm{LiBr}$ & 479.1 & $7.6(11)$ & 11.188 & 4.934 & 3.664 & 11.287 & 2.653 & 3.862 & 32.869 & $\mathrm{RS}$ \\
\hline LiI & 381.5 & $6.1(12)$ & 9.701 & 4.113 & 4.465 & 12.667 & 2.897 & 4.569 & 25.476 & $\mathrm{RS}$ \\
\hline $\mathrm{NaF}$ & 1062.7 & $11.5(13)$ & 12.275 & 7.416 & 1.821 & 4.170 & 2.227 & 3.156 & 61.943 & $\mathrm{RS}$ \\
\hline $\mathrm{NaCl}$ & 288.9 & $8.5(11)$ & 7.281 & 4.460 & 2.604 & 5.186 & 2.721 & 2.408 & 31.965 & $\mathrm{RS}$ \\
\hline $\mathrm{NaBr}$ & 179.2 & $7.1(14)$ & 6.357 & 3.589 & 3.003 & 5.728 & 2.886 & 3.554 & 26.127 & $\mathrm{RS}$ \\
\hline $\mathrm{NaI}$ & 115.6 & $5.9(12)$ & 5.439 & 2.920 & 3.582 & 6.659 & 3.125 & 4.078 & 20.181 & RS \\
\hline KF & 535.1 & $10.9(11)$ & 11.446 & 6.984 & 2.087 & 5.475 & 2.458 & 3.250 & 64.486 & $\mathrm{RS}$ \\
\hline $\mathrm{KCl}$ & 242.5 & $8.5(11)$ & 6.938 & 4.439 & 2.505 & 4.646 & 2.916 & 2.497 & 30.805 & $\mathrm{RS}$ \\
\hline $\mathrm{KBr}$ & 188.2 & $7.4(14)$ & 5.422 & 3.363 & 2.758 & 4.826 & 3.068 & 3.423 & 24.694 & $\mathrm{RS}$ \\
\hline KI & 278.9 & $6.2(11)$ & 4.595 & 2.720 & 3.121 & 5.017 & 3.296 & 3.848 & 18.896 & RS \\
\hline $\mathrm{RbF}$ & 612.1 & $10.4(11)$ & 10.795 & 6.025 & 2.220 & 5.949 & 2.537 & 5.313 & 67.228 & RS \\
\hline $\mathrm{RbCl}$ & 274.5 & $8.2(11)$ & 5.948 & 3.663 & 2.513 & 4.780 & 3.005 & 3.701 & 28.977 & $\mathrm{RS}$ \\
\hline $\mathrm{RbBr}$ & 154.9 & $7.2(15)$ & 4.218 & 2.708 & 2.724 & 4.798 & 3.152 & 4.383 & 23.829 & RS \\
\hline
\end{tabular}




\begin{tabular}{|c|c|c|c|c|c|c|c|c|c|c|}
\hline Name & $F_{\mathrm{b}}$ & $E_{\mathrm{g}}$ & $\omega_{\max }$ & $\omega_{\text {mean }}$ & $\varepsilon_{\mathrm{e}}$ & $\varepsilon_{\text {tot }}$ & $d_{\mathrm{NN}}$ & $\rho$ & $M$ & Structure \\
\hline $\mathrm{RbI}$ & 109.9 & $6.1(11)$ & 3.439 & 2.185 & 3.030 & 4.871 & 3.379 & 4.572 & 18.317 & $\mathrm{RS}$ \\
\hline $\mathrm{CsF}$ & 352.5 & $10.0(14)$ & 10.611 & 5.878 & 2.514 & 8.271 & 2.848 & 5.459 & 63.041 & $\mathrm{RS}$ \\
\hline $\mathrm{CsCl}$ & 348.2 & $8.3(16)$ & 6.388 & 3.771 & 3.140 & 7.207 & 3.253 & 5.276 & 41.100 & $\mathrm{CC}$ \\
\hline $\mathrm{CsBr}$ & 208.9 & $7.3(16)$ & 4.155 & 2.620 & 3.366 & 6.933 & 3.388 & 5.903 & 32.350 & $\mathrm{CC}$ \\
\hline CsI & 101.9 & $6.2(17)$ & 3.131 & 2.037 & 3.698 & 7.071 & 3.604 & 5.986 & 23.827 & $\mathrm{CC}$ \\
\hline \multicolumn{11}{|c|}{ Transition metal halides } \\
\hline $\mathrm{CuCl}$ & 181.6 & $3.4(18)$ & 8.465 & 4.724 & 5.481 & 7.202 & 2.275 & 4.533 & 70.639 & ZB \\
\hline $\mathrm{CuBr}$ & 117.4 & $2.9(19)$ & 5.763 & 3.819 & 6.541 & 8.140 & 2.401 & 5.588 & 60.827 & ZB \\
\hline $\mathrm{CuI}$ & 91.8 & $3.0(20)$ & 5.063 & 3.218 & 6.690 & 7.588 & 2.547 & 6.214 & 54.310 & $\mathrm{ZB}$ \\
\hline $\mathrm{AgF}$ & 198.7 & $5.0(21)$ & 7.844 & 3.769 & 5.253 & 9.901 & 2.424 & 7.398 & 90.178 & $\mathrm{RS}$ \\
\hline $\mathrm{AgCl}$ & 209.5 & $5.6(21)$ & 6.282 & 3.217 & 5.722 & 10.764 & 2.708 & 5.993 & 65.700 & $\mathrm{RS}$ \\
\hline $\mathrm{AgBr}$ & 101.6 & $4.8(21)$ & 4.345 & 2.559 & 6.800 & 12.397 & 2.823 & 6.930 & 59.375 & $\mathrm{RS}$ \\
\hline $\mathrm{AgI}$ & 92.2 & $2.8(22)$ & 4.041 & 2.598 & 5.827 & 7.151 & 2.775 & 5.927 & 38.940 & ZB \\
\hline \multicolumn{11}{|c|}{ Post-transition metal halides } \\
\hline $\mathrm{TlCl}$ & 255.2 & $3.4(23)$ & 5.956 & 2.468 & 6.293 & 33.041 & 3.022 & 9.374 & 43.934 & $\mathrm{CC}$ \\
\hline $\mathrm{TlBr}$ & 96.6 & $3.0(23)$ & 3.828 & 1.805 & 7.146 & 34.149 & 3.178 & 9.556 & 37.690 & $\mathrm{CC}$ \\
\hline TII & 141.2 & $2.8(24)$ & 2.914 & 1.415 & 8.203 & 38.088 & 3.388 & 9.184 & 31.069 & $\mathrm{CC}$ \\
\hline \multicolumn{11}{|c|}{ Alkaline earth metal chalcogenides } \\
\hline $\mathrm{BeS}$ & 479.8 & $7.4(25)$ & 20.290 & 12.470 & 5.412 & 7.141 & 2.063 & 2.522 & 99.251 & $\mathrm{ZB}$ \\
\hline $\mathrm{BeSe}$ & 512.8 & $5.5(26)$ & 18.632 & 10.059 & 6.111 & 7.607 & 2.191 & 4.509 & 81.905 & $\mathrm{ZB}$ \\
\hline $\mathrm{BeTe}$ & 346.2 & $2.8(27)$ & 16.730 & 8.844 & 7.498 & 8.395 & 2.384 & 5.438 & 62.557 & ZB \\
\hline $\mathrm{MgO}$ & 1609.9 & $7.8(28)$ & 20.642 & 12.255 & 3.090 & 9.350 & 2.067 & 3.790 & 171.100 & $\mathrm{RS}$ \\
\hline $\mathrm{MgS}$ & 211.5 & $2.7(29)$ & 11.852 & 6.932 & 5.509 & 14.013 & 2.558 & 2.798 & 82.086 & $\mathrm{RS}$ \\
\hline $\mathrm{MgSe}$ & 143.6 & $2.5(25)$ & 10.005 & 5.069 & 6.646 & 16.389 & 2.693 & 4.390 & 67.779 & $\mathrm{RS}$ \\
\hline $\mathrm{MgTe}$ & 141.9 & $2.3(30)$ & 8.641 & 4.723 & 5.622 & 7.813 & 2.749 & 3.942 & 38.352 & ZB \\
\hline $\mathrm{CaO}$ & 517.5 & $7.1(31)$ & 16.715 & 9.700 & 3.867 & 14.456 & 2.390 & 3.409 & 129.097 & $\mathrm{RS}$ \\
\hline $\mathrm{CaS}$ & 177.2 & $4.4(32)$ & 10.191 & 6.315 & 5.378 & 11.294 & 2.810 & 2.699 & 67.376 & $\mathrm{RS}$ \\
\hline $\mathrm{CaSe}$ & 117.9 & $3.9(33)$ & 7.968 & 4.727 & 6.014 & 11.677 & 2.922 & 3.960 & 56.621 & $\mathrm{RS}$ \\
\hline CaTe & 113.9 & $4.5(34)$ & 6.833 & 3.866 & 7.084 & 12.180 & 3.123 & 4.570 & 44.118 & $\mathrm{RS}$ \\
\hline $\mathrm{SrO}$ & 394.5 & $5.8(35)$ & 15.527 & 8.510 & 3.889 & 18.163 & 2.487 & 5.592 & 125.758 & $\mathrm{RS}$ \\
\hline $\mathrm{SrS}$ & 133.2 & $4.0(36)$ & 8.911 & 5.448 & 5.004 & 11.192 & 2.890 & 4.118 & 64.218 & $\mathrm{RS}$ \\
\hline $\mathrm{SrSe}$ & 115.2 & $4.6(35)$ & 6.208 & 3.936 & 5.558 & 11.277 & 2.998 & 5.131 & 53.812 & $\mathrm{RS}$ \\
\hline SrTe & 35.9 & $4.0(35)$ & 5.075 & 3.177 & 6.417 & 11.353 & 3.197 & 5.471 & 41.704 & RS \\
\hline $\mathrm{BaO}$ & 291.9 & $4.4(37)$ & 12.879 & 6.396 & 4.376 & 57.213 & 2.776 & 5.953 & 83.576 & $\mathrm{RS}$ \\
\hline $\mathrm{BaS}$ & 125.2 & $3.9(38)$ & 7.568 & 4.234 & 5.207 & 14.119 & 3.171 & 4.410 & 49.372 & $\mathrm{RS}$ \\
\hline $\mathrm{BaSe}$ & 76.9 & $3.6(38)$ & 5.031 & 3.065 & 5.737 & 13.333 & 3.269 & 5.141 & 42.702 & $\mathrm{RS}$ \\
\hline BaTe & 49.9 & $3.1(38)$ & 4.044 & 2.495 & 6.561 & 12.622 & 3.450 & 5.355 & 34.071 & $\mathrm{RS}$ \\
\hline \multicolumn{11}{|c|}{ Transition metal oxide } \\
\hline $\mathrm{MnO}$ & 231.2 & $3.8(39)$ & 13.490 & 5.458 & 15.471 & 17.258 & 2.243 & 5.217 & 156.370 & $\mathrm{RS}$ \\
\hline $\mathrm{FeO}$ & 187.9 & $2.4(40)$ & 13.301 & 5.675 & 10.367 & 13.941 & 2.168 & 5.853 & 189.990 & $\mathrm{RS}$ \\
\hline
\end{tabular}




\begin{tabular}{|c|c|c|c|c|c|c|c|c|c|c|}
\hline Name & $F_{\mathrm{b}}$ & $E_{\mathrm{g}}$ & $\omega_{\max }$ & $\omega_{\text {mean }}$ & $\varepsilon_{\mathrm{e}}$ & $\varepsilon_{\text {tot }}$ & $d_{\mathrm{NN}}$ & $\rho$ & $M$ & Structure \\
\hline $\mathrm{CoO}$ & 193.6 & $2.4(41)$ & 12.666 & 7.491 & 20.831 & 21.547 & 2.111 & 6.618 & 211.027 & $\mathrm{RS}$ \\
\hline $\mathrm{NiO}$ & 327.2 & $4.0(42)$ & 13.148 & 7.064 & 5.074 & 13.130 & 2.062 & 7.075 & 233.220 & RS \\
\hline \multicolumn{11}{|c|}{ Group IV semiconductor } \\
\hline $\mathrm{C}$ & 2624.0 & $5.5(18)$ & 40.513 & 29.674 & 5.765 & 5.765 & 1.523 & 3.661 & 460.524 & $\mathrm{ZB}$ \\
\hline $\mathrm{Si}$ & 84.2 & $1.1(43)$ & 15.401 & 9.872 & 13.225 & 13.225 & 2.330 & 2.394 & 94.414 & ZB \\
\hline $\mathrm{Ge}$ & 46.9 & $0.7(18)$ & 8.953 & 5.666 & 26.290 & 26.290 & 2.413 & 5.574 & 73.100 & $\mathrm{ZB}$ \\
\hline $\mathrm{SiC}$ & 419.5 & $2.4(18)$ & 28.015 & 17.998 & 6.934 & 10.280 & 1.864 & 3.336 & 222.949 & ZB \\
\hline $\mathrm{SiGe}$ & 52.6 & $0.9(44)$ & 9.894 & 6.278 & 14.827 & 14.831 & 2.367 & 4.092 & 84.105 & ZB \\
\hline \multicolumn{11}{|c|}{ Group III-V semiconductor } \\
\hline $\mathrm{BN}$ & 2062.1 & $6.4(18)$ & 38.333 & 26.001 & 4.564 & 6.725 & 1.546 & 3.622 & 397.099 & ZB \\
\hline BP & 428.8 & $2.4(18)$ & 24.772 & 16.617 & 9.186 & 9.340 & 1.939 & 3.090 & 172.929 & ZB \\
\hline BAs & 242.9 & $1.5(18)$ & 22.201 & 13.278 & 9.643 & 9.767 & 2.049 & 5.375 & 143.369 & ZB \\
\hline AlN & 881.7 & $4.9(45)$ & 25.516 & 15.834 & 4.545 & 8.280 & 1.863 & 3.418 & 205.352 & ZB \\
\hline AlP & 192.2 & $2.5(18)$ & 14.109 & 9.057 & 8.316 & 10.443 & 2.343 & 2.431 & 87.781 & $\mathrm{ZB}$ \\
\hline AlAs & 145.6 & $2.2(18)$ & 11.668 & 7.051 & 9.463 & 11.403 & 2.434 & 3.813 & 73.032 & $\mathrm{ZB}$ \\
\hline $\mathrm{AlSb}$ & 123.6 & $1.7(18)$ & 10.280 & 5.955 & 11.748 & 13.008 & 2.630 & 4.407 & 55.785 & ZB \\
\hline $\mathrm{GaN}$ & 691.7 & $3.2(45)$ & 23.649 & 13.767 & 5.687 & 9.791 & 1.866 & 6.946 & 228.398 & ZB \\
\hline $\mathrm{GaP}$ & 207.5 & $2.3(46)$ & 12.116 & 7.772 & 10.432 & 12.474 & 2.302 & 4.454 & 94.496 & ZB \\
\hline GaAs & 108.9 & $1.4(43)$ & 8.503 & 5.587 & 13.819 & 15.753 & 2.396 & 5.669 & 76.592 & ZB \\
\hline GaSb & 42.3 & $0.8(46)$ & 6.983 & 4.370 & 17.729 & 18.842 & 2.581 & 6.007 & 58.246 & ZB \\
\hline $\mathrm{InN}$ & 45.3 & $2.1(47)$ & 19.422 & 10.991 & 14.855 & 19.722 & 2.116 & 7.337 & 163.696 & ZB \\
\hline InP & 43.3 & $1.4(18)$ & 10.690 & 6.452 & 11.469 & 14.407 & 2.518 & 4.927 & 74.118 & ZB \\
\hline InAs & 10.6 & $0.4(46)$ & 7.008 & 4.391 & 18.136 & 21.030 & 2.601 & 5.814 & 61.556 & ZB \\
\hline $\mathrm{InSb}$ & 10.6 & $0.2(43)$ & 5.589 & 2.714 & 20.038 & 22.041 & 2.775 & 5.968 & 47.746 & ZB \\
\hline \multicolumn{11}{|c|}{ Group II-VI semiconductor } \\
\hline $\mathrm{ZnO}$ & 231.5 & $3.4(48)$ & 16.719 & 9.577 & 5.288 & 9.769 & 1.963 & 5.803 & 154.694 & ZB \\
\hline $\mathrm{ZnS}$ & 237.9 & $3.7(49)$ & 10.392 & 6.466 & 6.106 & 8.769 & 2.309 & 4.271 & 83.194 & ZB \\
\hline $\mathrm{ZnSe}$ & 150.2 & $2.8(49)$ & 7.231 & 4.709 & 7.439 & 10.038 & 2.426 & 5.451 & 68.612 & ZB \\
\hline $\mathrm{ZnTe}$ & 110.2 & $2.4(50)$ & 6.066 & 4.302 & 9.060 & 11.253 & 2.591 & 5.985 & 53.992 & ZB \\
\hline $\mathrm{CdO}$ & 181.9 & $0.8(18)$ & 13.301 & 6.724 & 7.930 & 19.227 & 2.347 & 8.246 & 155.366 & $\mathrm{RS}$ \\
\hline $\mathrm{CdS}$ & 78.2 & $2.5(51)$ & 9.090 & 5.203 & 6.565 & 9.702 & 2.519 & 4.876 & 65.201 & ZB \\
\hline $\mathrm{CdSe}$ & 15.3 & $1.7(52)$ & 5.953 & 3.784 & 8.588 & 11.662 & 2.626 & 5.696 & 55.323 & $\mathrm{ZB}$ \\
\hline $\mathrm{CdTe}$ & 20.2 & $1.5(51)$ & 4.786 & 3.064 & 9.165 & 11.712 & 2.781 & 6.020 & 44.747 & ZB \\
\hline $\mathrm{HgO}$ & 153.9 & $2.2(53)$ & 12.764 & 6.035 & 7.548 & 10.312 & 2.250 & 10.251 & 101.921 & $\mathrm{ZB}$ \\
\hline
\end{tabular}




\section{S5: ERROR ANALYSIS FOR PREDICTION MODELS}

In additional to the coefficient of determination $\left(R^{2}\right)$, systematic error analysis for the best performing prediction models with $\Omega$-dimensional descriptors are presented in terms of mean error (ME), mean absolute error (MAE), mean relative error (MRE), mean absolute relative error (MARE) and root mean square error (RMSE) as defined by

$$
\begin{gathered}
(\mathrm{ME})=\frac{1}{n} \sum_{i=1}^{n}\left(y_{i}-y_{i, \mathrm{pred}}\right), \quad(\mathrm{MAE})=\frac{1}{n} \sum_{i=1}^{n}\left|y_{i}-y_{i, \mathrm{pred}}\right| \\
(\mathrm{MRE})=\frac{1}{n} \sum_{i=1}^{n} \frac{y_{i}-y_{i, \mathrm{pred}}}{y_{i}}, \quad \quad(\mathrm{MARE})=\frac{1}{n} \sum_{i=1}^{n} \frac{\left|y_{i}-y_{i, \mathrm{pred}}\right|}{y_{i}} \\
(\mathrm{RMSE})=\sqrt{\frac{1}{n} \sum_{i=1}^{n}\left(y_{i}-y_{i, \mathrm{pred}}\right)^{2}}
\end{gathered}
$$

where $n$ is the number of data points, $y_{i}$ is the intrinsic dielectric breakdown field calculated by DFT framework, and $y_{i \text {,pred }}$ is predicted breakdown field using each prediction model (Figure S3). For the case of without cross-validation, errors obtained when the whole data set was used as the training set are reported. For the models with cross-validation, leave-10\%-out test set errors are reported.

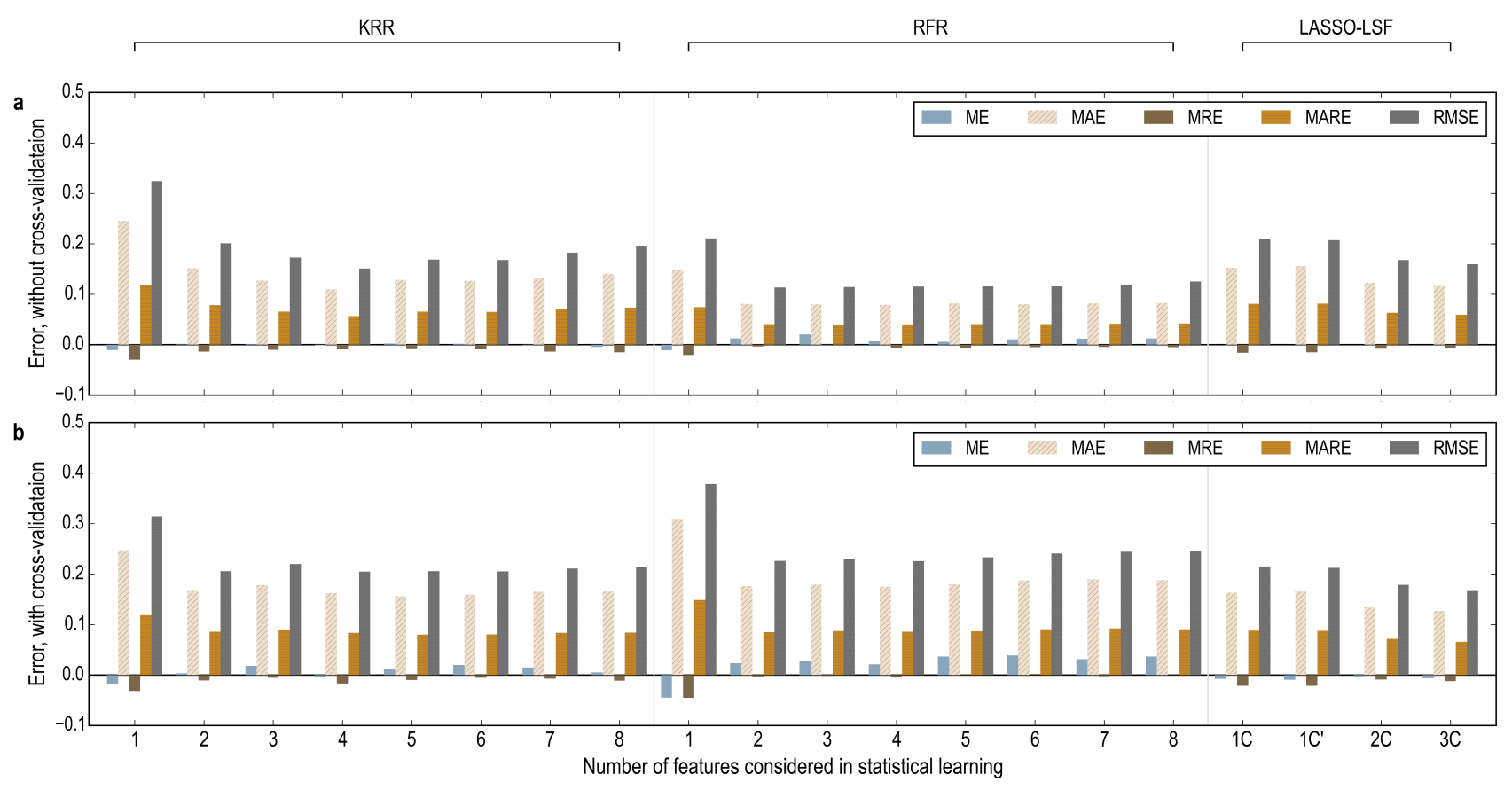

Figure S3. Error analysis for prediction models obtained by KRR, RFR and LASSO-LSF method. (a) Mean error (ME), mean absolute error (MAE), mean relative error (MRE), mean absolute relative error (MARE) and root mean square error (RMSE) of each model without cross-validation. (b) Errors of each model obtained using a leave-10\%-out cross-validation approach. In order to clarify that the LASSO-LSF model utilizes compound features, the number of features for this case is represented as $n \mathrm{C}$ with $n=1-3$. The LASSO-LSF procedure leads to two different 1-dimensional feature vector with equal predictive power, and these are indicated as $1 \mathrm{C}$ and $1 \mathrm{C}^{\prime}$. 


\section{S6: $\Omega$-DIMENSIONAL FEATURES WITH PREDICTION ERRORS}

The best $\Omega$-dimensional features are determined by exhaustively enumerating all possible features (i.e., 8 primary features in cases of KRR and RFR, and 36 down-selected compound features in case of LASSO), and ranking the models based on the performance of prediction in terms of $R^{2}$. Here we show 1- to 3dimensional features for the case of LASSO, because higher than 3-dimensional features are quite complex while the performance of the models built by those features does not have significant merit (c.f., $n$ dimensional features imply that the feature consists of $n$ primary or $n$ compound features). In order to achieve cross-validated models, 500 different random combinations of training $(90 \%)$ and test $(10 \%)$ sets are taken; then, the final performance is measured as an average over all 500 test runs. The performance of the prediction gradually improves as we go to higher dimensional features (Table S2).

Table S2. The best $\Omega$-dimensional features used for machine learning within kernel ridge regression (KRR), random forest regression (RFR) and least absolute shrinkage and selection operator (LASSO) methods. Mean error (ME), mean absolute error (MAE), mean relative error (MRE), mean absolute relative error (MARE) and root mean square error (RMSE) of cross-validatated models are listed together.

\begin{tabular}{|c|c|c|c|c|c|c|c|c|}
\hline Method & $\Omega$ & Features & $R^{2}$ & ME & MAE & MRE & MARE & RMSE \\
\hline \multirow[t]{8}{*}{ KRR } & 1 & $E_{\mathrm{g}}$ & 0.349 & -0.018 & 0.247 & -0.032 & 0.119 & 0.314 \\
\hline & 2 & $E_{\mathrm{g}}, \omega_{\max }$ & 0.713 & 0.004 & 0.168 & -0.010 & 0.086 & 0.206 \\
\hline & 3 & $E_{\mathrm{g}}, \omega_{\max }, \varepsilon_{\mathrm{e}}$ & 0.703 & 0.018 & 0.178 & -0.005 & 0.090 & 0.220 \\
\hline & 4 & $E_{\mathrm{g}}, \varepsilon_{\mathrm{e}}, d_{\mathrm{NN}}, M$ & 0.739 & -0.003 & 0.162 & -0.017 & 0.083 & 0.205 \\
\hline & 5 & $E_{\mathrm{g}}, \omega_{\max }, \varepsilon_{\mathrm{e}}, d_{\mathrm{NN}}, M$ & 0.743 & 0.012 & 0.156 & -0.010 & 0.080 & 0.206 \\
\hline & 6 & $E_{\mathrm{g}}, \omega_{\max }, \omega_{\text {mean }}, \varepsilon_{\mathrm{e}}, d_{\mathrm{NN}}, M$ & 0.743 & 0.020 & 0.159 & -0.005 & 0.081 & 0.206 \\
\hline & 7 & $E_{\mathrm{g}}, \omega_{\max }, \omega_{\text {mean }}, \varepsilon_{\mathrm{e}}, d_{\mathrm{NN}}, \rho, M$ & 0.699 & 0.015 & 0.165 & -0.007 & 0.084 & 0.211 \\
\hline & 8 & $E_{\mathrm{g}}, \omega_{\text {max }}, \omega_{\text {mean }}, \varepsilon_{\mathrm{e}}, \varepsilon_{\mathrm{tot}}, d_{\mathrm{NN}}, \rho, M$ & 0.663 & 0.005 & 0.166 & -0.011 & 0.084 & 0.214 \\
\hline \multirow[t]{8}{*}{$\mathrm{RF}$} & 1 & $E_{\mathrm{g}}$ & -0.045 & -0.045 & 0.310 & -0.045 & 0.149 & 0.379 \\
\hline & 2 & $E_{\mathrm{g}}, \omega_{\max }$ & 0.679 & 0.023 & 0.176 & -0.003 & 0.085 & 0.226 \\
\hline & 3 & $E_{\mathrm{g}}, \omega_{\text {max }}, \omega_{\text {mean }}$ & 0.674 & 0.028 & 0.179 & -0.002 & 0.087 & 0.229 \\
\hline & 4 & $E_{\mathrm{g}}, \omega_{\max }, \omega_{\text {mean }}, \rho$ & 0.675 & 0.021 & 0.175 & -0.005 & 0.086 & 0.226 \\
\hline & 5 & $E_{\mathrm{g}}, \omega_{\max }, \omega_{\text {mean }}, \varepsilon_{\mathrm{e}}, M$ & 0.658 & 0.037 & 0.180 & 0.002 & 0.087 & 0.233 \\
\hline & 6 & $E_{\mathrm{g}}, \omega_{\max }, \omega_{\text {mean }}, \varepsilon_{\mathrm{e}}, \varepsilon_{\mathrm{tot}}, M$ & 0.637 & 0.039 & 0.188 & 0.002 & 0.091 & 0.241 \\
\hline & 7 & $E_{\mathrm{g}}, \omega_{\mathrm{max}}, \omega_{\text {mean }}, \varepsilon_{\mathrm{e}}, \varepsilon_{\mathrm{tot}}, \rho, M$ & 0.621 & 0.032 & 0.190 & -0.002 & 0.093 & 0.244 \\
\hline & 8 & $E_{\mathrm{g}}, \omega_{\mathrm{max}}, \omega_{\text {mean }}, \varepsilon_{\mathrm{e}}, \varepsilon_{\mathrm{tot}}, d_{\mathrm{NN}}, \rho, M$ & 0.603 & 0.037 & 0.188 & 0.001 & 0.091 & 0.246 \\
\hline \multirow[t]{4}{*}{ LASSO } & $1 \mathrm{C}$ & $\left(E_{\mathrm{g}} \omega_{\max }\right)^{1 / 2}$ & 0.686 & -0.008 & 0.163 & -0.021 & 0.088 & 0.215 \\
\hline & $1 \mathrm{C}^{\prime}$ & $\omega_{\max } E_{\mathrm{g}}^{1 / 2} / \ln \left(\omega_{\text {mean }}\right)$ & 0.696 & -0.009 & 0.166 & -0.021 & 0.088 & 0.213 \\
\hline & $2 \mathrm{C}$ & $\omega_{\max }\left(E_{\mathrm{g}} \omega_{\text {mean }}\right)^{1 / 2}, d_{\mathrm{NN}}^{2} E_{\mathrm{g}}^{-1 / 2}$ & 0.786 & -0.003 & 0.134 & -0.009 & 0.072 & 0.179 \\
\hline & $3 \mathrm{C}$ & $\begin{array}{l}\omega_{\max }\left(E_{\mathrm{g}} \omega_{\text {mean }}\right)^{1 / 2}, \ln \left(E_{\mathrm{g}}\right) / d_{\mathrm{NN}}^{2}, \\
\ln \left(\omega_{\text {mean }}\right) /\left(\omega_{\max }{ }^{2} E_{\mathrm{g}}^{1 / 2}\right)\end{array}$ & 0.801 & -0.006 & 0.127 & -0.012 & 0.066 & 0.168 \\
\hline
\end{tabular}




\section{S7: PREDICTION MODELS FOUND BY LASSO-LSF WITH 1-DIMENSIONAL COMPOUND FEATURES}

In addition to the model with 1-dimensional feature composed of the band gap and phonon cutoff frequency as the 2 primary features (model ID 4 in Table S3, models with different nonlinear 1-dimensional features also show reasonable predictability (see Table S3). The band gap appears most frequently in those model formulas, followed by the phonon cutoff frequency and nearest neighbor distance.

Table S3. Details of model formulas for prediction of intrinsic dielectric breakdown field, obtained by LASSOLSF with 1 compound feature. $R^{2}$ and $R^{2} \mathrm{CV}$ are the coefficient of determination using entire dataset ( 82 cases) and test dataset ( 9 randomly chosen from 82 cases for cross-validation), respectively.

\begin{tabular}{clcc}
\hline \hline Model ID & Model formula & $R^{2}$ & $R_{\mathrm{CV}}^{2}$ \\
\hline 1 & $F_{\mathrm{b}}=43.554 \exp \left\{0.173 \omega_{\max } E_{\mathrm{g}}^{1 / 2} / \ln \left(\omega_{\text {mean }}\right)\right\}$ & 0.808 & 0.696 \\
2 & $F_{\mathrm{b}}=24.148 \exp \left\{0.240 \omega_{\max }\left(E_{\mathrm{g}} / \omega_{\text {mean }}\right)^{1 / 2}\right\}$ & 0.805 & 0.691 \\
3 & $F_{\mathrm{b}}=48.643 \exp \left\{0.735 \ln \left(E_{\mathrm{g}}\right) \ln \left(\omega_{\max }\right) / d_{\mathrm{NN}}{ }^{1 / 2}\right\}$ & 0.805 & 0.678 \\
4 & $F_{\mathrm{b}}=24.442 \exp \left\{0.315\left(E_{\mathrm{g}} \omega_{\max }\right)^{1 / 2}\right\}$ & 0.804 & 0.686 \\
5 & $F_{\mathrm{b}}=14.483 \exp \left\{0.975 \omega_{\max } /\left[\varepsilon_{\mathrm{e}}{ }^{1 / 2} \ln \left(\omega_{\text {mean }}\right)\right]\right\}$ & 0.798 & 0.692 \\
6 & $F_{\mathrm{b}}=46.326 \exp \left\{0.341 \omega_{\max }{ }^{1 / 2} \ln \left(E_{\mathrm{g}}\right)\right\}$ & 0.791 & 0.655 \\
7 & $F_{\mathrm{b}}=49.112 \exp \left\{18.278 \ln \left(E_{\mathrm{g}}\right) \ln \left(d_{\mathrm{NN}}\right) / d_{\mathrm{NN}}{ }^{3}\right\}$ & 0.789 & 0.651 \\
8 & $F_{\mathrm{b}}=54.462 \exp \left\{5.928 \ln \left(E_{\mathrm{g}}\right) / d_{\mathrm{NN}}{ }^{2}\right\}$ & 0.776 & 0.643 \\
9 & $F_{\mathrm{b}}=18.056 \exp \left\{1.709\left(\omega_{\max } / \varepsilon_{\mathrm{e}}\right)^{1 / 2}\right\}$ & 0.776 & 0.653 \\
10 & $F_{\mathrm{b}}=16.138 \exp \left\{3.064 E_{\mathrm{g}}{ }^{1 / 2} / d_{\mathrm{NN}}\right\}$ & 0.775 & 0.638 \\
\hline \hline
\end{tabular}

\section{S8: DATASET USED FOR VALIDATION OF PREDICTION MODELS}

For validation, we tested the robustness of our model by evaluating the intrinsic dielectric breakdown field of 4 materials not already included in our database, using DFT and the 3 phenomenological models. The materials considered are $\mathrm{Li}_{2} \mathrm{~S}, \mathrm{Na}_{2} \mathrm{~S}, \mathrm{SrCl}_{2}$, and $\mathrm{ZrO}_{2}$ that occur in the $F m-3 m$ structure (with 3 atoms per primitive cell). Experimental band gap for these materials in validation set is collected from previous studies while all other properties were computed here using DFT. The computed intrinsic dielectric breakdown field and the 8 primary features of the 4 materials are listed in Table S4.

Table S4. DFT-computed intrinsic dielectric breakdown field and basic properties of 4 compound materials used for validation of prediction models. $\left(F_{b}\right.$ : intrinsic dielectric breakdown field in $\mathrm{MV} / \mathrm{m}, E_{g}$ : experimental band gap in $\mathrm{eV}, \omega_{\max }$ : phonon cutoff frequency in $\mathrm{THz}, \omega_{\text {mean }}$ : mean phonon frequency in $\mathrm{THz}, \varepsilon_{\mathrm{e}}$ : electronic contribution of dielectric constant, $\varepsilon_{\mathrm{tot}}$ : total dielectric constant, $d_{\mathrm{NN}}$ : nearest neighbor distance in $\AA, \rho$ : density in $\mathrm{g} / \mathrm{cm}^{3}, M$ : bulk modulus in $\mathrm{GPa}$ )

\begin{tabular}{lrrrrrrrrr}
\hline \hline Name & $F_{\mathrm{b}}$ & $E_{\mathrm{g}}$ & $\omega_{\max }$ & $\omega_{\text {mean }}$ & $\varepsilon_{\mathrm{e}}$ & $\varepsilon_{\text {tot }}$ & $d_{\mathrm{NN}}$ & $\rho$ & $M$ \\
\hline $\mathrm{Li}_{2} \mathrm{~S}$ & 462.5 & $3.9(54)$ & 14.488 & 8.285 & 3.974 & 7.055 & 2.477 & 1.630 & 45.804 \\
$\mathrm{Na}_{2} \mathrm{~S}$ & 132.7 & $2.2(55)$ & 7.765 & 4.700 & 3.502 & 6.225 & 2.845 & 1.827 & 34.163 \\
$\mathrm{SrCl}_{2}$ & 293.4 & $7.5(56)$ & 5.822 & 3.359 & 3.120 & 7.103 & 3.051 & 3.009 & 43.746 \\
$\mathrm{ZrO}_{2}$ & 1253.0 & $5.8(57)$ & 22.231 & 12.828 & 5.963 & 37.541 & 2.150 & 6.686 & 262.801 \\
\hline \hline
\end{tabular}




\section{REFERENCES}

(1) Von Hippel, A. Electric Breakdown of Solid and Liquid Insulators. J. Appl. Phys. 1937, 8, 815-832.

(2) Fröhlich, H. Theory of Electrical Breakdown in Ionic Crystals. Proc. R. Soc. London, Ser. A 1937, 160, 230-241.

(3) Fröhlich, H. Theory of Dielectric Breakdown. Nature 1943, 151, 339-340.

(4) Fröhlich, H. On the Theory of Dielectric Breakdown in Solids. Proc. R. Soc. London, Ser. A 1947, 188, 521-532.

(5) Hastie, T.; Tibshirani, R.; Friedman, J. The Elements of Statistical Learning: Data Mining, Inference, and Prediction, 2nd ed.; Springer: New York, 2009.

(6) Muller, K. R.; Mika, S.; Ratsch, G.; Tsuda, K.; Scholkopf, B. An Introduction to Kernel-based Learning Algorithms. IEEE Trans. Neural Netw. 2001, 12, 181-201.

(7) Hofmann, T.; Scholkopf, B.; Smola, A. J. Kernel Methods in Machine Learning. Ann. Statist. 2008, 36, 1171-1220.

(8) Tibshirani, R. Regression Shrinkage and Selection via the Lasso. J. Roy. Stat. Soc. B 1996, 58, 267-288.

(9) Breiman, L. Random Forests. Mach. Learn. 2001, 45, 5-32.

(10) Sun, Y.; Boggs, S. A.; Ramprasad, R. The Intrinsic Electrical Breakdown Strength of Insulators from First Principles. App. Phys. Lett. 2012, 101, 132906.

(11) Poole, R. T.; Jenkin, J. G.; Liesegang, J.; Leckey, R. C. G. Electronic Band Structure of the Alkali Halides. I. Experimental Parameters. Phys. Rev. B 1975, 11, 5179-5189.

(12) Baldini, G.; Bosacchi, B. Optical Properties of Alkali-Halide Crystals. Phys. Rev. 1968, 166, 863-870.

(13) Fröhlich, D.; Staginnus, B. New Assignment of the Band Gap in the Alkali Bromides by Two-Photon Spectroscopy. Phys. Rev. Lett. 1967, 19, 496-498.

(14) Haensel, R.; Keitel, G.; Peters, G.; Schreiber, P.; Sonntag, B.; Kunz, C. Photoemission Measurement on $\mathrm{NaCl}$ in the Photon Energy Range 32-50 eV. Phys. Rev. Lett. 1969, 23, 530-532.

(15) Brown, F. C.; Gähwiller, C.; Fujita, H.; Kunz, A. B.; Scheifley, W.; Carrera, N. Extreme- Ultraviolet Spectra of Ionic Crystals. Phys. Rev. B 1970, 2, 2126-2138.

(16) Teegarden, K.; Baldini, G. Optical Absorption Spectra of the Alkali Halides at 10K. Phys. Rev. 1967, 155, 896-907.

(17) DiStefano, T. H.; Spicer, W. E. Photoemission from CsI: Experiment. Phys. Rev. B 1973, 7, 1554-1564.

(18) Landolt-Börnstein - Group III Condensed Matter, Numerical Data and Functional Relationships in Science and Technology; Martienssen, W.; Madelung, O.; Hellwege, K.-H., Ed; Springer-Verlag: Berlin Heidelberg, 2003.

(19) Ves, S.; Glötzel, D.; Cardona, M.; Overhof, H. Pressure Dependence of the Optical Properties and the Band Structure of the Copper and Silver Halides. Phys. Rev. B 1981, 24, 3073-3085.

(20) Sankapal, B.; Goncalves, E.; Ennaoui, A.; Lux-Steiner, M. Wide Band Gap P-type Windows by CBD and SILAR Methods. Thin Solid Films 2004, 451-452, 128-132.

(21) Kunz, A. B. Electronic Structure of AgF, AgCl, and AgBr. Phys. Rev. B 1982, 26, 2070-2075. 
(22) Micic, O. I.; Meglic, M.; Lawless, D.; Sharma, D. K.; Serpone, N. Semiconductor photophysics. 5. Charge Carrier Trapping in Ultrasmall Silver Iodide Particles and Kinetics of Formation of Silver Atom Clusters. Langmuir 1990, 6, 487-492.

(23) Bachrach, R. Z.; Brown, F. C. Exciton-Optical Properties of TlBr and TlCl. Phys. Rev. B 1970, 1, 818831.

(24) Fujii, A.; Takiyama, K.; Nakahara, J.; Kobayashi, K. Magneto-Optical Absorption in Thallous Iodide of CsCl Structure. J. Phys. Soc. Jpn 1977, 42, 525-528.

(25) Ropp, R. Encyclopedia of the Alkaline Earth Compounds; Elsevier Science, 2013.

(26) Wilmers, K.; Wethkamp, T.; Esser, N.; Cobet, C.; Richter, W.; Cardona, M.; Wagner, V.; Lugauer, H.; Fischer, F.; Gerhard, T.; Keim, M. Ellipsometric Studies of $\mathrm{Be}_{\mathrm{x}} \mathrm{Zn}_{1-\mathrm{x}} \mathrm{Se}$ Between $3 \mathrm{eV}$ and $25 \mathrm{eV}$. Phys. Rev. B 1999, 59, 10071-10075.

(27) Waag, A.; Litz, T.; Fischer, F.; Lugauer, H.-J.; Baron, T.; Schll, K.; Zehnder, U.; Gerhard, T.; Lunz, U.; Keim, M.; Reuscher, G.; Landwehr, G. In Advances in Solid State Physics 37; Helbig, R., Ed.; Advances in Solid State Physics; Springer Berlin Heidelberg, 1998; Vol. 37; pp 43-66.

(28) Roessler, D. M.; Walker, W. C. Electronic Spectrum and Ultraviolet Optical Properties of Crystalline MgO. Phys. Rev. 1967, 159, 733-738.

(29) Takabatake, T.; Tanaka, T.; Bando, Y.; Fujii, H.; Takeda, N.; Ishikawa, M.; Oguro, I. Magnetic and Structural Transitions in $\mathrm{CeRh}_{2} \mathrm{Sb}_{2}$. Physica B 1997, 230-232, 223-225.

(30) Parker, S. G.; Reinberg, A. R.; Pinnell, J. E.; Holton, W. C. Preparation and Properties of $\operatorname{Mg}_{x} Z_{1-x} T e$. J. Electrochem. Soc. 1971, 118, 979-983.

(31) Whited, R.; Flaten, C. J.; Walker, W. Exciton Thermoreflectance of MgO and CaO. Solid State Commun. 1973, 13, 1903-1905.

(32) Radhakrishna, S.; Daud, A. Solid State Materials; Springer: Berlin Heidelberg, 2013.

(33) Shameem Banu, I. B.; Kalpana, G.; Palanivel, B.; Shenbagaraman, P.; Rajagopalan, M.; Yousuf, M. Ab Initio Electronic Band Structure Calculations for Calcium Monochalco- genides. Int. J. Mod. Phys. B 1998, 12, 1709-1717.

(34) Khokhlov, D. Lead Chalcogenides: Physics and Applications; Optoelectronic properties of semiconductors and superlattices; Taylor \& Francis, 2002.

(35) Joshi, N. Photoconductivity: Art: Science \& Technology; Optical Science and Engineering; Taylor \& Francis, 1990.

(36) Piqu, A.; Auyeung, R.; Qadri, S.; Kim, H.; Justus, B.; Huston, A. Growth of Epitaxial Doped Strontium Sulfide Thin Films by Pulsed Laser Deposition. Thin Solid Films 2000, 377-378, 803-808.

(37) Köhler, H.; Fabbicius, A. Galvanomagnetic Properties of $\mathrm{Bi}_{2} \mathrm{Se}_{3}$ with Free Carrier Den- sities below $5 \times 10^{17} \mathrm{~cm}^{-3}$. Phys. Status Solidi B 1975, 71, 487-496.

(38) Zollweg, R. J. Optical Absorption and Photoemission of Barium and Strontium Oxides, Sulfides, Selenides, and Tellurides. Phys. Rev. 1958, 111, 113-119.

(39) Iskenderov, R. N.; Drabkin, I. A.; Emel'anova, L. T.; Ksendzov, Y. M. Absorption Spectrum of MnO Single Crystals, Fiz. Tverd. Tela 1968, 10, 2573-2574.

(40) Gramsch, S.; Cohel, R.; Savrasov, S. Structure, Metal-insulator Transitions, and Mag- netic Properties of FeO at High Pressures. Am. Mineral. 2003, 88, 257-261. 
(41) Cheetham, A. K.; Hope, D. A. O. Magnetic Ordering and Exchange Effects in the Anti- ferromagnetic Solid Solutions $\mathrm{Mn}_{x} \mathrm{Ni} 1-{ }_{x} \mathrm{O}$. Phys. Rev. B 1983, 27, 6964-6967.

(42) Hüfner, S.; Osterwalder, J.; Riesterer, T.; Hulliger, F. Photoemission and Inverse Photoemission Spectroscopy of NiO. Solid State Commun. 1984, 52, 793-796.

(43) Blatt, F. Modern Physics; Physics series; McGraw-Hill, 1992.

(44) People, R. Indirect Band Gap of Coherently Strained $\mathrm{Ge}_{\mathrm{x}} \mathrm{Si}_{1-\mathrm{x}}$ Bulk Alloys on $<001>$ Silicon Substrates. Phys. Rev. B 1985, 32, 1405-1408.

(45) Sitar, Z.; Paisley, M.; Ruan, J.; Choyke, J.; Davis, R. Luminescence and Lattice Parameter of Cubic Gallium Nitride. J. Mat. Sci. Lett. 1992, 11, 261-262.

(46) Thornton, S.; Rex, A. Modern Physics for Scientists and Engineers; Saunders golden sunburst series; Saunder's College Pub., 1993.

(47) Jenkins, D. W.; Hong, R.-D.; Dow, J. D. Band Structure of InN. Superlattices Microstruct. 1987, 3, 365369.

(48) Mang, A.; Reimann, K.; Rbenacke, S. Band gaps, Crystal-field Splitting, Spin-orbit Coupling, and Exciton Binding Energies in $\mathrm{ZnO}$ under Hydrostatic Pressure. Solid State Commun. 1995, 94, 251-254.

(49) Madelung, O. Semiconductors: Other than Group IV Elements and III-V Compounds (Data in Science and Technology); Data in Science and Technology; Springer-Verlag, 1992.

(50) Madelung, O. Semiconductors: Data Handbook; Springer Berlin Heidelberg, 2004.

(51) Singh, J. Physics of Semiconductors and Their Heterostructures; Electrical engineering series; McGrawHill, 1993.

(52) Shan, W.; Walukiewicz, W.; Ager, J. W.; Yu, K. M.; Wu, J.; Haller, E. E. Pressure Dependence of the Fundamental Band-gap Energy of CdSe. Appl. Phys. Lett. 2004, 84, 67-69.

(53) Landolt-Börnstein - Group III Condensed Matter $41 B$ (II-VI and I-VII Compounds; Semimagnetic Compounds), Mercury oxide (HgO) crystal structure, physical properties; Madelung, O.; Rössler, U.; Schulz, M., Ed.; Springer-Verlag, 1999.

(54) Isikaku-Ironkwe, O. Is Lithium Sulfide a MgB2-like Superconductor? arXiv preprint arXiv:1205.4051 2012.

(55) Lopes, R.; Spencer, J. Io After Galileo: A New View of Jupiter's Volcanic Moon; Springer Praxis Books; Springer Berlin Heidelberg, 2007.

(56) Sugiura, C. $K$-x-ray Spectra and Electronic Band Structures of $\mathrm{MgCl}_{2}, \mathrm{CaCl}_{2}, \mathrm{SrCl}_{2}$, and $\mathrm{BaCl}_{2}$. Phys. Rev. B 1974, 9, 2679-2683.

(57) Bhattacharya, P.; Fornari, R.; Kamimura, H. Comprehensive Semiconductor Science and Technology; Elsevier Science, 2011. 